\title{
Facies de patch reefs en la Formación Picún Leufú (límite Jurásico/Cretácico) en la región de Zapala, Cuenca Neuquina
}

\author{
Claudia ARMELLA ${ }^{1}$, Nora CABALERI ${ }^{1} \&$ Héctor A. LEANZA²
}

\begin{abstract}
${ }^{1}$ CONICET-UBA, Pabellón INGEIS - Ciudad Universitaria. 1428 Buenos Aires. Argentina. armella@ingeis.uba.ar. ${ }^{2}$ CONICET-SEGEMAR, Av. Julio A. Roca 651, 10ㅜㅜ piso. 1322 Buenos Aires. Argentina.

Abstract: Patch reefs facies in the Picún Leufú Formation (Jurassic/Cretaceous boundary) in the Zapala region, Neuquén Basin, Argentina. In the locality of Cerrito Caracoles, situated in the nearby of Zapala city, in the western central region of the Neuquén province, Argentina, the basal part of the Picún Leufú Formation (Jurassic/Cretaceous boundary) crops out. The facies and microfacies association analysis points out to an inner shelf margin environment, with development of well preserved patch reefs. The description of these bodies (coral and algae bafflestone, bryozoan bafflestone and algal boundstone), and of a low relief mound reef (skeletal algal packstone) are presented in this paper. The associated facies are represented by low energy deposits (mudstone), bar complexes (sandy skeletal packstone and skeletal packstone) and channels (skeletal intraclast floatstone and intraclast skeletal ooidal floatstone).
\end{abstract}

Key words: Sedimentology, Facies/Microfacies analysis, Patch reefs, Jurassic/Cretaceous boundary, Picún Leufú Formation, Neuquén Basin.

Resumen: En el cerrito Caracoles, ubicado en las cercanías de Zapala, en el sector centro occidental de la provincia del Neuquén, Argentina, aflora el tramo basal de la Formación Picún Leufú (límite Jurásico/Cretácico). El análisis de la asociación de facies y microfacies indica características de margen de la plataforma interna, con desarrollo de patch reefs bien preservados. En este trabajo se presenta la descripción de estos cuerpos (bafflestone de corales y algas, bafflestone de briozoarios y boundstone algal) y de un montículo (mound reef) de bajo relieve (packstone bioclástico algal). Las facies asociadas están representadas por depósitos de baja energía (mudstone), complejos de barras (packstone biosilicoclástico y packstone bioclástico) y canales (floatstone biointraclástico y floatstone intrabiooidal).

Palabras clave: Sedimentología, análisis de facies/microfacies, patch reefs, límite Jurásico/Cretácico, Formación Picún Leufú, Cuenca Neuquina.

\section{INTRODUCCIÓN}

La zona de estudio se encuentra en el ámbito del Engolfamiento Neuquino, al sudeste de la Cuenca Neuquina. El cerrito Caracoles está situado a la vera de la ruta $\mathrm{n}^{\circ} 22,13 \mathrm{~km}$ al noroeste de Zapala, en la comarca conocida como Los Catutos, en el sector centro-occidental de la provincia del Neuquén (Fig. 1). Durante el Jurásico superior y el Cretácico inferior el ambiente marino permaneció continuo en toda la cuenca, se restableció la conexión marina con el Pacífico y se inició una etapa de sedimentación carbonática representada en esta región por depósitos de calizas blanquecinas, frecuentemente bioturbadas y bancos coquinoides de la Formación Picún Leufú. El motivo de esta contribución es presentar el resultado del análisis de facies y microfacies de la secuencia basal de la Formación Picún Leufú, aflorante en el cerrito Caracoles y la interpretación del modelo paleoambiental local.

\section{MATERIALES Y MÉTODOS}

El trabajo de campo consistió en el relevamiento y muestreo en detalle del perfil expuesto en el cerrito Caracoles. En esta localidad la sucesión carbonática alcanza $22 \mathrm{~m}$ de potencia y está compuesta por calizas de color blanco, frecuentemente bioturbadas y bancos coquinoides. Los trabajos de gabinete comprenden el estudio petrográfico de cortes delgados y superficies pulidas, en todos los casos orientados con respecto a la base del estrato. Las muestras se tiñeron con Alizarina S Roja para detectar el contenido de dolomita y con ferricianuro de potasio para la identificación cualitativa de hierro. Para el análisis de microfacies se siguió la clasificación de Dunham (1962), modificada por Embry \& Klovan (1971) y Flügel (1982). La descripción e interpretación del modelo paleoambiental se realizó según los criterios de Wilson (1975), Read (1985), Reading (1996) y Wright \& Burchette (1996). 


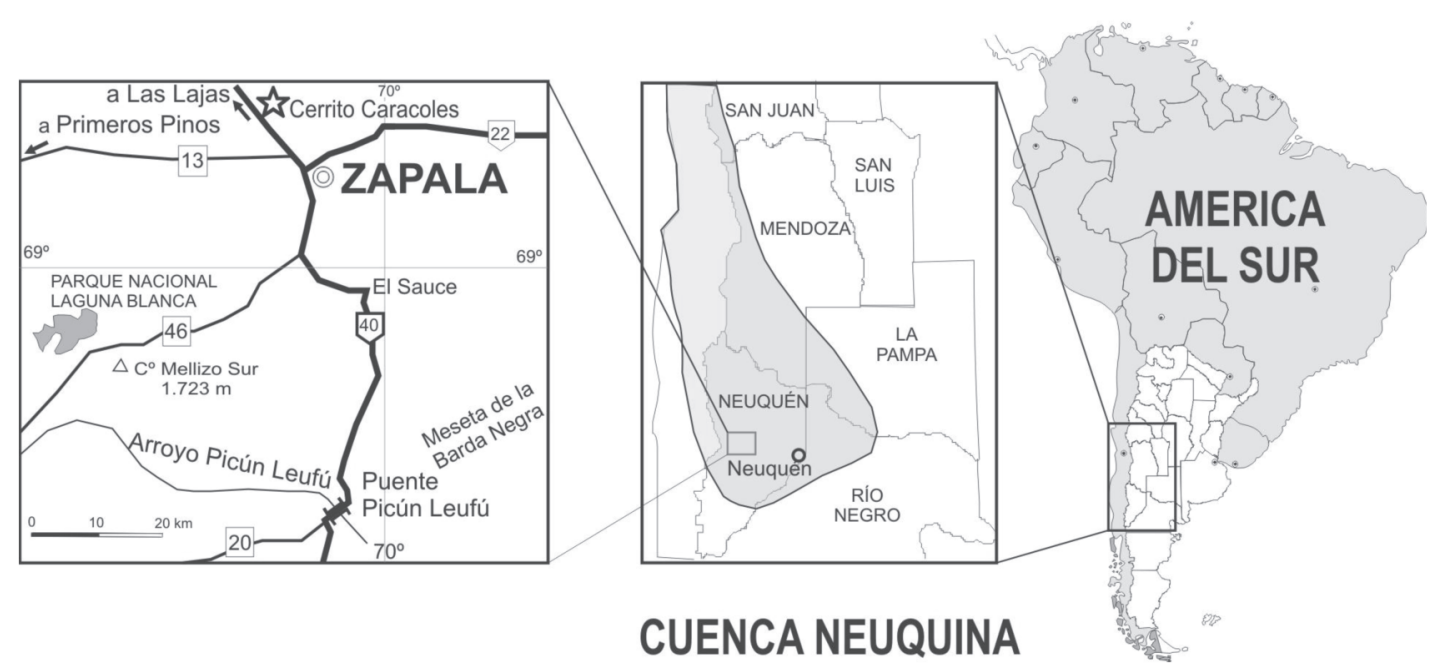

Fig. 1. Ubicación de la localidad de cerrito Caracoles y zona de estudio.

\section{ESTRATIGRAFÍA}

La estratigrafía de la cuenca Neuquina se caracteriza por el desarrollo de una espesa secuencia sedimentaria que se acumuló desde el Triásico Superior hasta el Paleógeno temprano. Dentro de esta columna, que alcanza los $7.000 \mathrm{~m}$ de espesor, los depósitos del Jurásico y el Cretácico son mundialmente conocidos por la excelencia de sus afloramientos.

Las variaciones eustáticas se consideran el principal factor de control en la evolución geodinámica de la región, aunque en muchos casos se sobreimponen a fenómenos tectónicos locales (Legarreta \& Gulisano, 1989; Legarreta \& Uliana, 1991).

Las sedimentitas marinas -con breves interrupciones continentales- depositadas durante el Jurásico Superior y el Cretácico Inferior son conocidas colectivamente como Grupo Mendoza (Stipanicic et al., 1968).

En el Tithoniano inferior tardío y medio (Zonas de Mendozanus, Zitteli y Proximus) se produjo la acumulación de sedimentos finos, silicoclásticos y carbonáticos, ricos en materia orgánica (lutitas y margas bituminosas con concreciones calcáreas), que caracterizan a la Formación Vaca Muerta (Weaver, 1931). Esta unidad fue el resultado de una importante transgresión que causó una marcada expansión del área de acumulación marina, que superó en extensión a las unidades jurásicas infrayacentes. En los alrededores del cerrito Caracoles, la Formación Vaca Muerta presenta aproximadamente $300 \mathrm{~m}$ de potencia, y en su tramo superior se destaca la intercalación de un conjunto de calizas de naturaleza litográfica de unos 70 a $90 \mathrm{~m}$ de espesor conocida como Miembro Los Catutos (Leanza \& Zeiss, 1990).

Posteriormente, en el Tithoniano medio tardío al Berriasiano, las condiciones de sedimentación resultaron favorables para la depositación de facies carbonáticas someras, nominadas por Leanza (1973), como Formación Picún Leufú, esta unidad se desarrolla estratigráficamente entre las Formaciones Vaca Muerta y Bajada Colorada (Roll, en Fossa Mancini et al., 1938). Se encuentra bien expuesta en la margen sudoriental de la cuenca (Carbonate Shelf según Legarreta \& Uliana, 1991), siendo interpretada como resultado de depositación en un régimen de mar alto (Highstand Systems Tract) por Leanza \& Hugo (1997), Armella et al., (1999, 2007) y Spalletti et $a l .$, (2000). Se le asignó una edad comprendida entre el Tithoniano medio alto (Zona de Internispinosum) y el Berriasiano inferior (Zona de Noduliferum) por contenido de fauna de amonites (Leanza 1973, 1981; Leanza \& Zeiss 1990; Leanza \& Hugo, 1978, 1997). De tal modo, en esta región de la cuenca puede detectarse el límite Jurásico/ Cretácico. En la Fig. 2 se representa el perfil columnar general de la comarca de Los Catutos, con la ubicación estratigráfica del tramo basal de la Formación Picún Leufú en la localidad del cerrito Caracoles, basado en el trabajo de Leanza \& Zeiss (1990).

Con referencia al paleoambiente de la Formación Picún Leufú, Armella et al. (2007) determinaron mediante análisis de facies/microfacies y de su contenido faunístico las condiciones de sedimentación en ambiente marino, con aguas tem- 
pladas poco profundas, bien oxigenadas, en una plataforma carbonática, con pendiente suave y formación de complejo de barras, que se desarrolla ampliamente en la región sudoriental de la citada cuenca. A nivel geológico regional de conocen en la comarca de Los Catutos los mapeos de detalle de Leanza \& Zeiss (1990) y el regional correspondiente a la Hoja geológica Zapala (Leanza et al., 2001)

\section{DESCRIPCIÓN DE LOS PATCH REEFS Y PALEAMBIENTES RELACIONADOS}

El resultado de los análisis de microfacies y facies de los afloramientos de la Formación Picún Leufú permitió reconstruir las condiciones de sedimentación carbonática durante el límite Jurásico/Cretácico. El modelo paleoambiental corresponde a una extensa plataforma, con predominio de facies de plataforma interna, disectada por canales progradantes, y facies de lagoon con bancos y barras. En el área de cerrito Caracoles, donde sólo está expuesto el tramo basal de la unidad (Fig. 3), la asociación de facies y microfacies (véase Tabla 1) refleja condiciones marinas más profundas, correspondientes al margen de la plataforma interna, sobre la que se desarrolló un complejo de barras y canales, con facies de baja energía, de ambiente protegido (lagoon) y formación de patch reefs (Armella et al, 1999, 2007).

En el registro geológico los patch reefs se reconocen principalmente por su geometría lenticular y por la presencia de organismos constructores (corales, bivalvos y algas). La paleoecología de las comunidades bioconstructoras está influenciada por una variedad de controles externos, tales como el aporte sedimentario, la energía del medio, oxigenación, suministro de la luz y cantidad de nutrientes (Hallock \& Schlager, 1986; Carannante et al., 1995; Dupraz \& Strasser, 2002, Götz et al., 2005). Los patch reefs son estructuras pequeñas, normalmente rodeados por facies de lagoon, con crecimiento vertical determinado por la altura del nivel del mar, debajo de la base de ola de buen tiempo. Sin embargo, durante bajamar, su superficie puede quedar expuesta. Se diferencian de las barras carbonáticas por carecer estos cuerpos de un armazón bioconstruido y son de mayor escala.

Las facies de barras están representadas por cuerpos sigmoidales acrecionales progradantes, formados en los niveles inferiores del perfil por packstone biosilicoclástico, compuesto por fragmentos de bioclastos de bivalvos, frecuentemente orientados (Trigonia carinata, Exogyra couloni, Ostrea lotenoensis, O. minos, Cercomya sp.), amonites (Substeueroceras sp., Corongoceras sp.), y espinas de equinodermos, silicoclastos, intra-

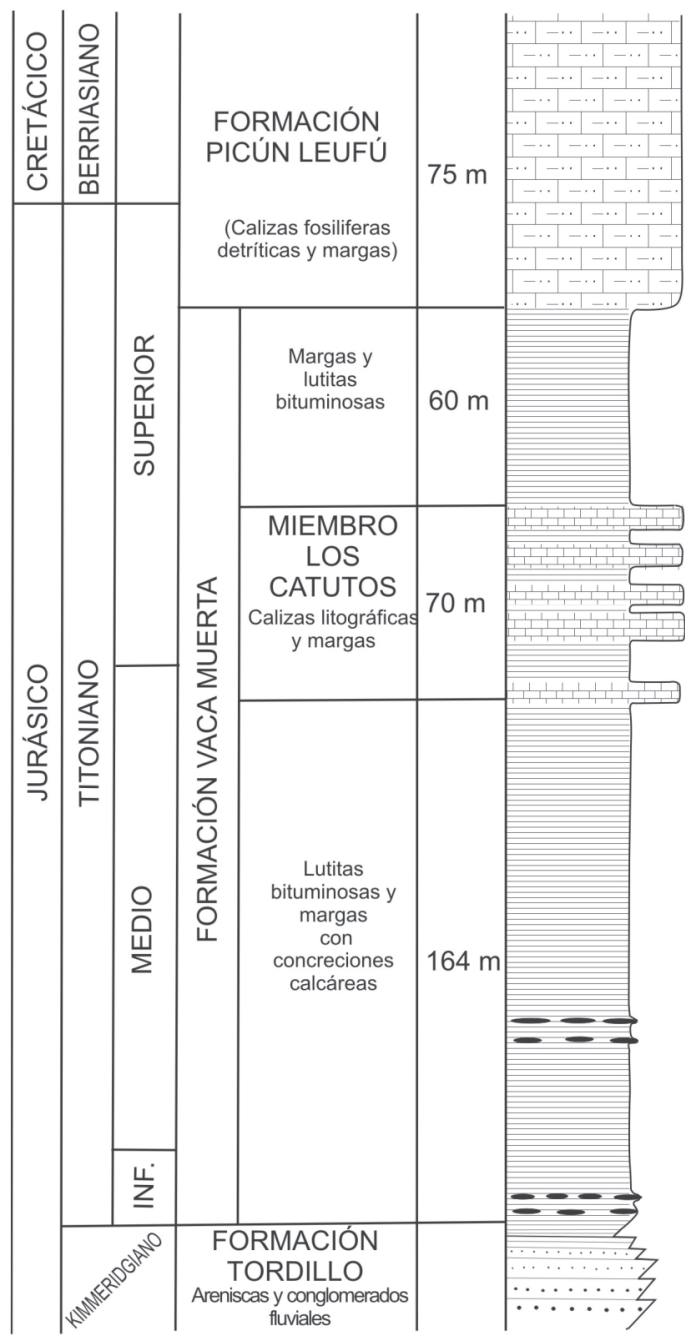

Fig. 2. Perfil estratigráfico-litológico columnar general de la comarca de Los Catutos, con ubicación estratigráfica del tramo basal de la Formación Picún Leufú.

clastos y escasos ooides superficiales y radiales. La asociación de partículas señala condiciones de acumulación de alta energía, sometidas al constante movimiento del oleaje de buen tiempo en áreas próximas al margen de la plataforma. Sobre esta facies se formaron bancos de packstone bioclástico, amalgamados y con estratificación entrecruzada del tipo hummocky, compuestos por abundantes valvas muy retrabajadas y orientadas de bivalvos (ostréidos y Lucina sp.), restos de amonites (Pseudosubplanites grandis), briozoarios, gastrópodos muy fragmentados, espinas de 


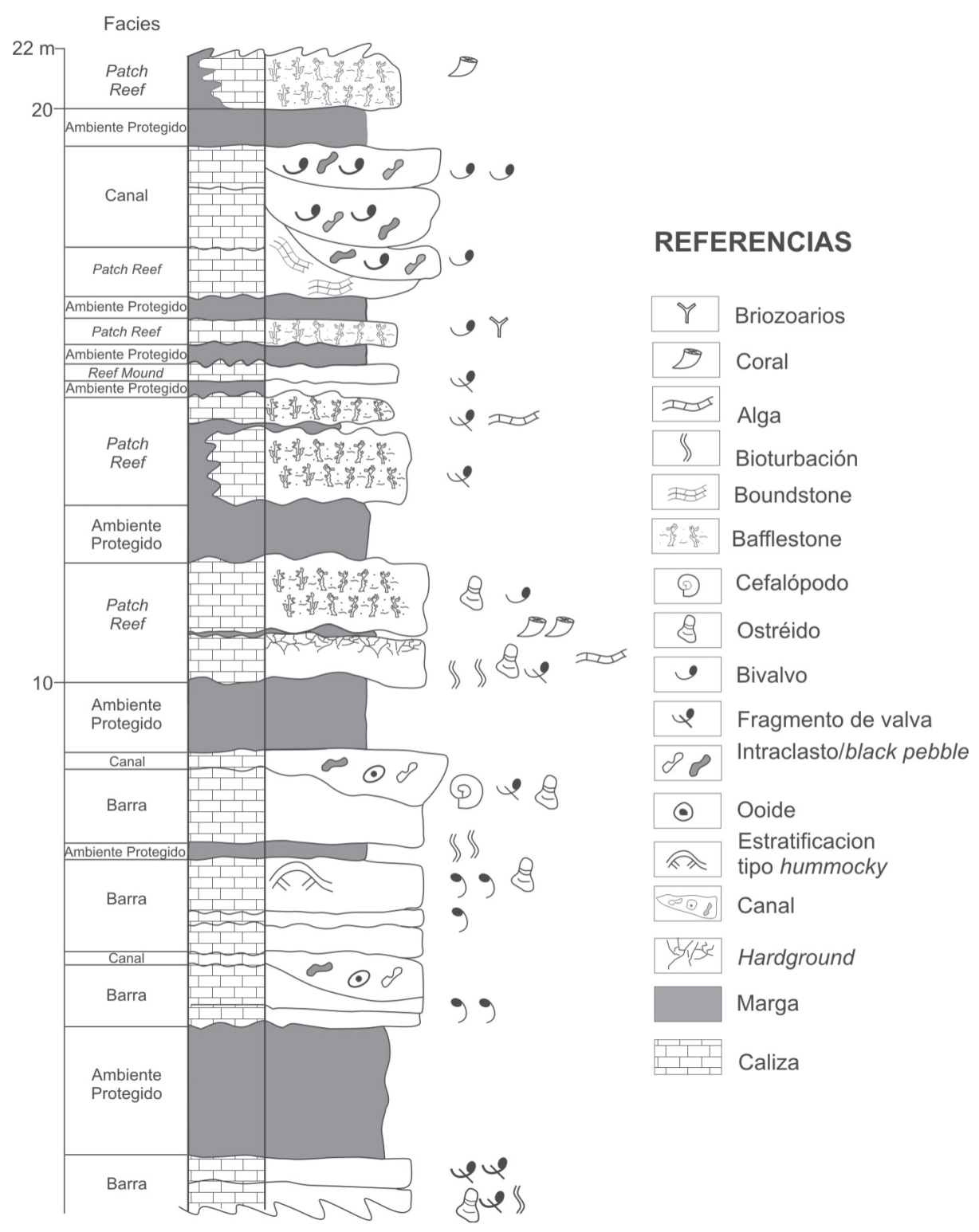

Fig. 3. Perfil de detalle del tramo basal de la Formación Picún Leufú, aflorante en la localidad del cerrito Caracoles.

equinodermos y foraminíferos indeterminados. Se interpreta que estos bancos son el resultado de depósitos formados por las olas de tormentas.

Las barras están disectadas por canales que trasportaron materiales provenientes de zonas costeras hacia el mar abierto, representados por niveles lenticulares de floatstone biointraclástico.

En la facies de baja energía (lagoon) los fangos carbonáticos, con mezcla de arena fina, se depositaron en ambiente submareal y protegido, representados por niveles de mudstone margoso. Este sustrato fue el hábitat propicio para pelecípodos infaunales (Pholadomya sp.), encontrados en posición de vida. La asociación con icnofacies del tipo Thalassinoides y Planolites, señala condiciones de depositación en aguas calmas, someras y no afectadas por la ola de buen tiempo (Pemberton et al., 1992; Buatois et al., 2002). En el lagoon los organismos coloniales y bioconstructores (corales, bivalvos y algas) encon- 

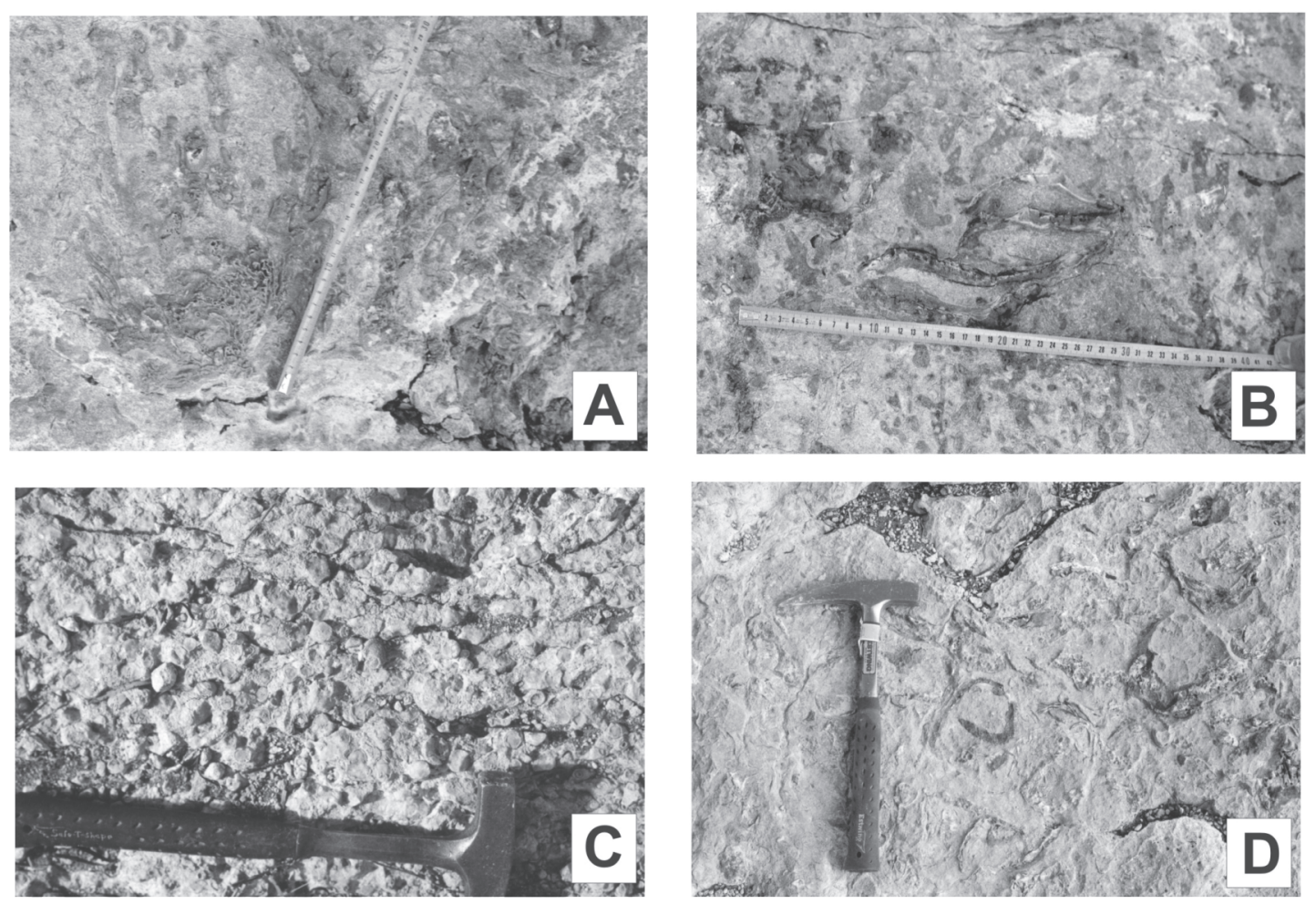

Fig. 4. Facies de patch reefs en la Formación Picún Leufú en la localidad del cerrito Caracoles. A, Bafflestone de corales y algas, desplegando colonias de corales ramosos. B, Bafflestone de corales y algas del nivel superior, mostrando detalles de algas filoides y restos de corales. C, Bafflestone de briozoarios, en vista tomada en planta del afloramiento, mostrando briozoarios en posición de vida con relleno de packstone/grainstone oobioclástico. D, Boundstone algal, con algas filoides en wackestone bioclástico.

traron condiciones favorables para su crecimiento: saturación de luz, aguas poco profundas y nutrientes (Wray, 1977; James, 1984). En la base de la mitad superior del perfil (Fig. 3) se reconoció un nivel de hardground, con abundantes galerías y perforaciones transversales a la estratificación (Thalassinoides y Skolithos) que proporcionó el sustrato firme en el se fijaron los organismos coloniales.

Las facies de patch reef se distinguen en la sección medio superior del perfil del cerrito Caracoles y presentan pobre diversidad de organismos bioconstructores. Se diferenciaron tres tipos de patch reef, bafflestone de corales y algas, bafflestone de briozoarios y boundstone algal, y un montículo de bajo relieve (reef mound), formado por packstone bioclástico algal. Se presentan dispersos en el lagoon, en las áreas marginales de la plataforma interna, detrás de las barras, formando lentes o montículos hemisféricos con un diseño de planta elíptico o irregular.
Los cuerpos de bafflestone de corales y algas (Figs. 4, A-B) son domales, discontinuos, de aproximadamente $1 \mathrm{~m}$ de espesor y $2 \mathrm{~m}$ de longitud. Las colonias de corales de finas ramificaciones formaron una estructura abierta y en los niveles superiores del cuerpo predominan algas filoides. Entre la fábrica bioconstruida quedó retenido el material de relleno (packstone/grainstone bioclástico) compuesto por restos de briozoarios, bivalvos y corales solitarios, bioclastos de ostrácodos, gastrópodos, foraminíferos, espinas de equinodermos y algas. La fábrica abierta y el crecimiento de corales con ramificaciones delicadas señalan poca resistencia a la acción del oleaje. Esta estructura organosedimentaria se desarrolla bajo condiciones de alta tasa de sedimentación que favorece la acumulación de sedimentos durante el crecimiento vertical de los organismos (James, 1984). Las colonias de corales y algas se acrecionaron verticalmente hasta alcanzar el nivel del mar. 
Tabla 1. Síntesis de las microfacies analizadas en la localidad de cerrito Caracoles.

\begin{tabular}{|c|c|c|c|}
\hline MICROFACIES & COMPOSICIÓN & FÁBRICA & $\begin{array}{l}\text { CONDICIONES } \\
\text { AMBIENTALES }\end{array}$ \\
\hline Mudstone margoso & $\begin{array}{l}\text { Matriz: micrita peloidal con } \\
\text { minerales de hierro, arcilla y } \\
\text { materia orgánica. } \\
\text { Cemento: esparita inters- } \\
\text { ticial. } \\
\text { Partículas: silicoclastos. }\end{array}$ & $\begin{array}{l}\text { Bivalvos en posición de vida. } \\
\text { (Pholadomya sp). } \\
\text { Icnofósiles: Thalassinoides y } \\
\text { Planolites. }\end{array}$ & $\begin{array}{l}\text { Ambiente protegido } \\
\text { (lagoon), aguas poco } \\
\text { profundas }\end{array}$ \\
\hline $\begin{array}{c}\text { Packstone } \\
\text { biosilicoclástico }\end{array}$ & $\begin{array}{l}\text { Matriz: principalmente mi- } \\
\text { crítica. } \\
\text { Cemento: mosaico de espa- } \\
\text { rita, calcita magnesiana. } \\
\text { Partículas: bioclastos, bi- } \\
\text { valvos, silicoclastos, intra- } \\
\text { clastos, ooides superficiales y } \\
\text { radiales esparíticos. }\end{array}$ & $\begin{array}{l}\text { Fragmentos de conchillas po- } \\
\text { bremente seleccionados, bi- } \\
\text { valvos orientados. }\end{array}$ & $\begin{array}{c}\text { Barra } \\
\text { Alta energía }\end{array}$ \\
\hline Packstone bioclástico & $\begin{array}{l}\text { Matriz: micrita/microspa-rita. } \\
\text { Cemento: esparita y dolo- } \\
\text { mita. } \\
\text { Partículas: bioclastos, valvas } \\
\text { de bivalvos y amonites, intra- } \\
\text { clastos, silicoclastos. }\end{array}$ & $\begin{array}{l}\text { Bancos amalgamados, con es- } \\
\text { tratificación hummocky, Val- } \\
\text { vas orientadas. }\end{array}$ & $\begin{array}{c}\text { Bancos } \\
\text { Alta energía } \\
\text { Influencia de tormentas }\end{array}$ \\
\hline $\begin{array}{l}\text { Floatstone } \\
\text { Biointraclástico }\end{array}$ & $\begin{array}{l}\text { Matriz: bioclástica con mi- } \\
\text { crita. } \\
\text { Cemento: esparita/micros- } \\
\text { parita. } \\
\text { Particulas: Bioclastos, in- } \\
\text { traclastos, cortoides, black } \\
\text { pebbles, silicoclastos, ooides }\end{array}$ & $\begin{array}{l}\text { Partículas pobremente selec- } \\
\text { cionadas. } \\
\text { Fragmentos retrabajados, } \\
\text { conchillas de bivalvos con la } \\
\text { convexidad hacia arriba. } \\
\text { Bioturbación. }\end{array}$ & $\begin{array}{c}\text { Canales } \\
\text { Corrientes mareales } \\
\text { canalizadas entre las } \\
\text { barras. }\end{array}$ \\
\hline $\begin{array}{l}\text { Floatstone } \\
\text { intrabiooidal }\end{array}$ & $\begin{array}{l}\text { Matriz: micrita. } \\
\text { Cemento: esparita. } \\
\text { Partículas: restos arreci-fales } \\
\text { y de matas algales, bioclastos, } \\
\text { intraclastos, cortoides, black } \\
\text { pebbles, ooides } \\
\text { Partículas pobremente selec- } \\
\text { cionadas. }\end{array}$ & $\begin{array}{l}\text { Partículas pobremente selec- } \\
\text { cionadas. }\end{array}$ & $\begin{array}{l}\text { Canales próximos al } \\
\text { talud. }\end{array}$ \\
\hline $\begin{array}{l}\text { Bafflestone de corales } \\
\text { y algas }\end{array}$ & $\begin{array}{l}\text { Matriz: micrita. } \\
\text { Cemento: esparita. } \\
\text { Partículas: bioclastos, cal- } \\
\text { ciesferas, ooides concén- } \\
\text { tricos, silicoclastos }\end{array}$ & $\begin{array}{l}\text { Colonias ramosas de corales } \\
\text { en posición de vida, forman- } \\
\text { do estructuras delicadas. } \\
\text { Algas filoides. }\end{array}$ & $\begin{array}{c}\text { Patch reef } \\
\text { Energía moderada }\end{array}$ \\
\hline $\begin{array}{l}\text { Bafflestone de } \\
\text { briozoarios }\end{array}$ & $\begin{array}{l}\text { Matriz: micrita. } \\
\text { Cemento: crecimiento con- } \\
\text { céntrico de esparita y sílice } \\
\text { rodeando a los organismos } \\
\text { coloniales. } \\
\text { Partículas: bioclastos, con- } \\
\text { chillas de bivalvos, intra- } \\
\text { clastos, ooides, silicoclastos. }\end{array}$ & $\begin{array}{l}\text { Colonias de briozoarios en } \\
\text { posición de vida, formando } \\
\text { una estructura orgánica poco } \\
\text { resistente a la acción de las } \\
\text { olas. }\end{array}$ & $\begin{array}{c}\text { Patch reef } \\
\text { Aguas muy someras, } \\
\text { baja energía. }\end{array}$ \\
\hline Boundstone algal & $\begin{array}{l}\text { Matriz: micrita. } \\
\text { Cemento: escaso } \\
\text { Partículas: bioclastos, algas } \\
\text { filoides. }\end{array}$ & $\begin{array}{l}\text { Algas incrustantes en el techo } \\
\text { del boundstone }\end{array}$ & Patch reef \\
\hline $\begin{array}{l}\text { Packstone bioclástico } \\
\text { algal }\end{array}$ & $\begin{array}{l}\text { Matriz: micrita } \\
\text { Cemento: esparita } \\
\text { Partículas: bioclastos, intra- } \\
\text { clastos, silicoclastos. }\end{array}$ & $\begin{array}{l}\text { Partículas muy fragmentadas } \\
\text { y mal seleccionadas. }\end{array}$ & $\begin{array}{c}\text { Reef mound } \\
\text { Afectado por la acción } \\
\text { de olas, con episodios } \\
\text { de exposición y de } \\
\text { erosión. }\end{array}$ \\
\hline
\end{tabular}


El segundo tipo de patch reefs, caracterizado por bafflestone de briozoarios (Fig. 4, C) forma cuerpos domales aislados de $1,5 \mathrm{~m}$ de espesor y 3 $\mathrm{m}$ de longitud, en los que se diferencia un nivel basal, de packstone bioclástico, sobre el que se desarrolla una incipiente estructura orgánica en posición de vida representada por colonias de briozoarios. Dentro de esta fábrica abierta se reconocieron restos de bivalvos dispersos, intraclastos, ooides y silicoclastos que forman el relleno sedimentario. Hacia el techo del cuerpo la estructura es más cerrada y el material ligante es un packstone/grainstone oobioclástico con ostrácodos; las partículas están recubiertas por filamentos algales. La buena preservación de los briozoarios en posición de vida, sumado a la abundancia de bioclastos sugieren condiciones de aguas calidas y claras en un ambiente submareal, debajo de la base de olas de buen tiempo (James, 1984).

$\mathrm{El}$ boundstone algal (Fig. 4, D) forma lentes extendidas, de $0.60 \mathrm{~m}$ de espesor y $2 \mathrm{~m}$ de longitud, compuestas por tres niveles. La base está caracterizada por wackestone bioclástico pobremente seleccionado, con bioclastos muy fragmentados y orientados. En el sector medio se reconocieron niveles de algas filoides y en el superior algas incrustantes que forman la cubierta del boundstone. Estas algas son frecuentes en ambientes de lagoon con alta tasa de sedimentación e indican depositación dentro de la zona fótica, en aguas someras. (Wray, 1977; Flügel, 1991). Entre estos cuerpos se identificaron niveles canalizados de floatstone intrabiooidal, formados bajo condiciones de mayor energía que las facies asociadas e interpretados como flujos de alta densidad (Mutti, 1992) cercanos al talud que recibieron el aporte de material de diferentes áreas de la plataforma (Armella et al., 2007).

El cuerpo de packstone bioclástico algal forma un montículo de bajo relieve, está compuesto por partículas mal seleccionadas y muy fragmentadas, con recubrimientos de micrita y óxidos de hierro (cortoides). Predominan bioclastos de bivalvos, secciones de algas dasicladáceas y escamas de peces. Este montículo se habría desarrollado cercano al nivel del mar, afectado por la acción del oleaje.

\section{CONCLUSIONES}

El límite Jurásico/Cretácico está representado en el ámbito sudoriental de la cuenca Neuquina por la amplia plataforma carbonática de la Formación Picún Leufú.

En la localidad de cerrito Caracoles sólo está expuesta la sección basal de la unidad, que corresponde a las facies marginales del subambiente de plataforma interna, cercanas al talud, caracterizadas por un complejo de barras, canales, facies de lagoon y patch reefs.

Se diferenciaron tres tipos de patch reefs (bafflestone de corales y algas, bafflestone de briozoarios y boundstone algal). Los organismos coloniales y bioconstructores crecieron en un ambiente de baja energía, formando cuerpos lenticulares o montículos de bajo relieve dispersos en el lagoon. El crecimiento vertical de los organismos estuvo limitado por la altura del nivel del mar, el grado de oxigenación, suministro de la luz y cantidad de nutrientes. Las fábricas abiertas de estos cuerpos y las estructuras delicadas de corales y briozoarios, señalan condiciones de aguas someras, claras, de baja a moderada energía del medio, en un ambiente submareal, debajo de la base de olas de buen tiempo.

\section{AGRADECIMIENTOS}

Los autores desean expresar su agradecimiento al Sr. Gabriel Giordanengo (INGEIS, Buenos Aires) por la confección de los dibujos y a la Dirección General de Minería de la provincia del Neuquén por facilitarnos el transporte en el área de trabajo. Este trabajo fue financiado por el Consejo Nacional de Investigaciones Científicas y Técnicas (CONICET), PEI 0087/97 y PIP 5222.

\section{BIBLIOGRAFIA}

Armella, C., N. Cabaleri \& H.A. Leanza. 1999. Paleoambientes de la Formación Picún Leufú (límite Jurásico-Cretácico) en su localidad tipo, Cuenca Neuquina, Argentina. $5^{\circ}$ Simposio sobre o Cretáceo do Brasil. $1^{\circ}$ Simposio sobre el Cretácico de América del Sur, Serra Negra, Brasil, pp. 357-358.

- 2007. Tidally dominated, rimmed-shelf facies of the Picún Leufú Formation (Jurassic/Cretaceous boundary) in southwest Gondwana, Neuquén Basin, Argentina. Cretaceous Research, 28 (6): 961-979.

Buatois, L., G. Mangano \& F.G. Aceñolaza. 2002. Trazas Fósiles: Señales de Comportamiento en el Registro Estratigráfico. Edición Especial, Museo Paleontológico Egidio Feruglio $n^{\circ} 2$, Argentina, $382 \mathrm{pp}$.

Carannante, G., A. Cherchi \& L. Simone. 1995. Chlorozoan versus foramol lithofacies in Upper Cretaceous rudist limestones. Palaeogeography, Palaeoclimatology, Palaeoecology 119: 137-154.

Dunham, R.J. 1962. Classification of carbonate rocks according to depositional texture. En: W. E. Ham (ed), Classification of Carbonate Rocks, American Association of Petroleum Geologists, Memoir 1: 108-121.

Dupraz, C. \& A. Strasser. 2002. Nutritional modes in coral-microbialite reefs (Jurassic, Oxfordian, Switzerland): evolution of trophic structure as a response to environmental change. Palaios 17: 449-471.

Embry A.F \& J.E. Klovan. 1971. A late Devonian reef tract on north-eastern Banks Island, Northwest 
Territories, Bulletin of Canadian Petroleum Geology 19: 730-781.

Flügel, E. 1982. Microfacies Analysis of Limestones. Springer-Verlag, Berlin, 633 pp.

- 1991. Triassic and Jurassic marine calcareous algae: a critical review. En: R. Riding (ed.), Calcareous Algae and Stromatolites, Springer-Verlag, Berlin, pp. 481-504.

Fossa Mancini, E., E. Feruglio \& J.C. Yussen de Campana. 1938. Una reunión de geólogos de YPF y el problema de la terminología estratigráfica, Boletín de Informaciones Petroleras 14(171): 1-67.

Götz, S., H. Löser \& D.U. Schmid. 2005. Reef development on a deepening platform: two Early Cretaceous coralgal patch reefs (Catí, Llácova Formation, eastern Spain) compared, Cretaceous Research 26: 64-881

Hallock, P. \& W. Schlager. 1986. Nutrient excess and the demise of coral reefsand carbonate platforms. Palaios 1: 389-398.

James, N.P. 1984. Reefs. En: R.G. Walker (ed.), Facies Models. Geological Association of Canada, Ottawa, pp. 229-244.

Leanza, H.A. 1973. Estudio sobre los cambios facies de los estratos limítrofes Jurásico-Cretácicos entre Loncopué y Picún Leufú, provincia del Neuquén, República Argentina, Revista de la Asociación Geológica Argentina 28 (1): 97-132.

- 1981. The Jurassic/Cretaceous boundary beds in west central Argentina and their ammonite zones, Neues Jahrbuch für Geologie und Paläontologie, Abhandlungen 161: 62-92.

Leanza, H.A \& C.A. Hugo. 1978. Sucesión de amonites y edad de la Formación Vaca Muerta y sincrónicas entre los paralelos $35^{\circ}$ y $40^{\circ}$ l. s. Cuenca NeuquinaMendocina. Revista de la Asociación Geológica Argentina, 32 (4): 248-264.

- $\quad$ 1997. Hoja Geológica 3969-III, Picún Leufú, provincias del Neuquén y Río Negro. Programa Nacional de Cartas Geológicas a escala 1: 250.000. Instituto de Geología y Recursos Minerales, SEGEMAR, Buenos Aires, Boletín 218: 1-135.

Leanza, H.A., C.A. Hugo \& D. Repol. 2001. Hoja geológica 3969 - I, Zapala provincia del Neuquén. Programa Nacional de Cartas Geológicas de la República Argentina a escala 1 : 250.000. Instituto de Geología y Recursos Minerales. SEGEMAR. Boletín 275: 1-128.

Leanza, H.A. \& A. Zeiss. 1990. Upper Jurassic lithographic limestone from Argentina (Neuquén Basin): stratigraphy and fossils. Facies 22: 169-186.
Legarreta, L. \& C. Gulisano. 1989. Análisis estratigráfico secuencial de la Cuenca Neuquina (Triásico superior-Terciario inferior). Cuencas sedimentarias Argentinas. Facultad de Ciencias Naturales, Universidad Nacional de Tucumán. Correlación Geológica, Serie 6: 221-243.

Legarreta, L. \& M.A. Uliana. 1991. Jurassic/Cretaceous marine oscillations and geometry of a back-arc basin fill, central Argentine Andes. En: D.I.M. McDonald (ed.), Sedimentation, Tectonics and Eustacy, International Association of Sedimentologists, Special Publication 12: 429-450.

Mutti, E. 1992. Turbidite Sandstones, Instituto di Geologia, Università di Palma; AGIP SA, Milan, 275 pp.

Pemberton, S.G., J.A. MacEachern, \& R.W. Frey. 1992. Trace fossil facies models: environmental and alostratigraphic significance. In: R.G. Walker \& N.P. James (eds.), Facies Models: Response to Sea Level Changes. Geological Association of Canada, Ottawa, pp. 47-72.

Read, J.F. 1985. Carbonate platform facies models, American Association of Petroleum Geologists, Bulletin 69: $1-21$

Reading, H.G. 1996. Sedimentary Environments: Processes, Facies and Stratigraphy (Third edition), Blackwell Science, Oxford, 688 pp.

Spalletti, L.A., J.R. Franzese, S.D. Matheos \& E. Schwarz. 2000. Sequence stratigraphy of a tidally dominated carbonate-siliciclastic ramp; the Tithonian-Early Berriasian of the southern Neuquén Basin, Argentina, Journal of the Geological Society 157: 433-446.

Stipanicic, P.N., F. Rodrigo, O.L. Baulíes \& C.G. Martínez, 1968. Las formaciones presenonianas del denominado Macizo Nordpatagónico y regiones adyacentes. Revista de la Asociación Geológica Argentina 23: 367-388.

Weaver, Ch. 1931. Paleontology of the Jurassic and Cretaceous of west central Argentina. University of Washington, Seattle, Memoir 1, 469 pp.

Wilson, J.L. 1975. Carbonate Facies in Geologic History, Springer-Verlag, Berlin-Heidelberg, $471 \mathrm{pp}$.

Wray, J.L. 1977. Calcareous Algae. Developments in Paleontology and Stratigraphy, 4, Elsevier, Amsterdam, $185 \mathrm{pp}$.

Wright V.P. \& T.P. Burchette. 1996. Shallow-water carbonate environments. En: H.G. Reading (ed.), Sedimentary Environments: Processes, Facies and Stratigraphy, Blackwell Scientific publications, Oxford, pp. 325-394. 